\title{
ON THE DYNAMICS OF THE LAGEOS-I SPIN VECTOR: HIGH PRECISION DIRECT OBSERVATIONS AND COMPARISONS TO THEORETICAL MODELING
}

\author{
D. CURRIE, K. KISSELL, P. AVIZONIS AND D. WELLNITZ \\ Department of Physics, University of Maryland \\ College Park, Maryland, USA
}

\begin{abstract}
LAGEOS I is a high-density geodetic satellite launched by NASA on 4 May 1976 (Johnson et al., 1976). Using a network of laser ranging stations, GSFC/NASA has maintained extremely accurate information on the orbital motions of LAGEOS I, and the later LAGEOS II and on the time-dependent evolution of their orbital parameters. The development of short-pulse laser ranging systems, and better models for atmospheric refractive effects, have dramatically improved the ability to locate the spacecraft or measure geodetic position and terrestrial crustal motion, but these systems do not measure the satellite rotational motion or gyroscopic effects, thought initially unimportant. Primarily as a result of technical strides in orbit determination, it is now recognized that the spin-motion is critical to understanding weak but important interactions with the environment.
\end{abstract}

\section{Introduction}

It is because of the potential for another scientific use of the LAGEOS spacecraft, a use in modern physics quite distinct from their use in modern geodesy, that we seek to understand several subtle effects caused entirely by the spin rotation of these nearly spherically symmetric spacecraft. Because of the very precise orbital information, the LAGEOS satellites can be very effective tools in performing a precise measurement of one of the most interesting consequences of General Relativity, in the concept advanced for the "LAGEOS III" experiment to measure the Lense-Thirring effect (Ciufolini, 1986; Ciufolini, 1994). An important requirement for the success of this experiment proves to be a precise knowledge of the orientation of the spin axes of the satellites (Ries et al., 1988). Recently such a body of

I. M. Wytrzyszczak, J. H. Lieske and R. A. Feldman (eds.),

Dynamics and Astrometry of Natural and Artificial Celestial Bodies, 341, 1997.

(C) 1997 Kluwer Academic Publishers. Printed in the Netherlands. 
directly observed data on the orientation of the spin axis of LAGEOS I has been obtained (Currie et al., 1992; Currie et al., 1995).

From the point-of-view of the basic physics governing a spinning artificial satellite, the LAGEOS satellite is a nearly ideal candidate for developing a verifiable theoretical model of the rotational dynamics as influenced by the Lorenz and tidal forces. Complicating these analyses is the need to account for another low-level force, a study of "photon rocket thrust", that is, the total response of the spacecraft to the combined radiation fields of the Sun (visible wavelength) and of the Earth (IR wavelengths), when combined with the surface thermal emission (IR), on the orbital elements of the satellite. However, in order to address the question of the adequacy of a given theoretical model, precise direct observations of the orientation of the spin axis must be available. A body of such data has been obtained, from our observations and from various other sources, and analyzed within a University of Maryland program conducted over the past five years. The techniques used in these observations, the analysis methods and the results of this program are presented. This approach has determined the orientation of the spin axis generally to an accuracy much better than one degree and the rotation rate to much better than $1 \%$.

Finally, in order to increase the time interval for the comparison to theory, data from other sources are being analyzed. Other observations using various remote sensing techniques performed in the late 1970s and early 1980s by the Lincoln Laboratories of MIT (Rork, 1996) and other groups (Kissell, 1992) are on hand but not yet analyzed. Candidate systems and new procedures proposed to obtain observations in the future are also addressed.

\section{Background}

The role of the orientation and spin axis of LAGEOS plays a critical role in two areas. The first is the improved analysis for geodetic and long-term Earth physics phenomenon. The effects of spin orientation lead to anomalous orbit-perturbing forces, which in turn, can lead to errors in terrestrial analyses. The infamous "once-per-rev" effect appears now to be explainable as a spin orientation effect (Rubincam, Currie and Robbins, 1997). In addition, in terms of the LAGEOS orbit itself, the orientation effects cause the largest uncertainties in the analysis of the Lense-Thirring effect involved in the proposed LAGEOS III experiment (Ciufolini, 1986; Ries, 1989). The ability to measure the spin-axis motion is, therefore, crucially important. Finally, it represents a very interesting classical physics problem to study the effects of electromagnetic and tidal forces on a relatively compact and clean satellite of fairly well-known internal structure. 
Comparisons are being made between the observational data and predictions of various theoretical models and the results of various indirect techniques in an area of active research. This includes the predictions of the models of Bertotti and Iess (Bertotti and Iess, 1991), Miller and Habib (Habib et al., 1994), and Farinella and Vokrouhlicky (Farinella et al., 1995). Comparisons have been made between our direct observations and, first, the indirect information obtained by the theoretical analysis of observations of the orbital acceleration (Rubincam, 1990), and, second, the change in the orbital acceleration caused by the passage of the satellite through the shadow of the Earth (Ries et al., 1993; Robbins et al., 1994).

We will address the measurements of LAGEOS I made at Maryland, and less frequent measurements made at other sites, to obtain a spin-axis history with accuracies at the few degrees to a fraction of a degree level.

\section{Method of Measurement}

The method used for these measurements is the observation by groundbased telescopes of a specular (Fresnel) reflection of the Sun from the front face of one of the 426 corner prisms on LAGEOS. These flashes typically have a width, in satellite rotation, of a quarter of a degree. The peak of the glint will approximate the surface normal of the prism being aligned with the bisector of the observer-LAGEOS-Sun phase angle. Thus it is typical for one component of the spin-axis determination to have that accuracy. The accuracy of the other component involves the evolution of these measurements and may be 3 to 15 times less precise. Measurements from two passes within a short time allows the fractional degree accuracy to be reached in both components. Since the corner prisms are installed in numerous latitudinal belts (Figure 1), it is necessary to be able to ascribe the operative prism to a given latitude. This is a key part of the bookkeeping of the observations when the spacecraft rotational period is in minutes rather than seconds.

The techniques used for the detection and timing of glints range from early millisecond-accuracy photoelectric photometry, required when spin rates are high $(30-100 \mathrm{rpm})$ and the glint durations short $(1-3 \mathrm{msec})$, to video-rate intensified cameras or CCD's and now, with LAGEOS I, to visual recognition and timing. For the last decade, video cameras with frame-time encoding have proved adequate when the glint durations extend over several frames. The cameras were used behind telescopes of 0.3 to $1.2-\mathrm{m}$ aperture.

\section{Analysis Technique}

The current data analysis scheme could be described as a dynamic extrapolation of a slowly varying function in which a correct choice of the under- 


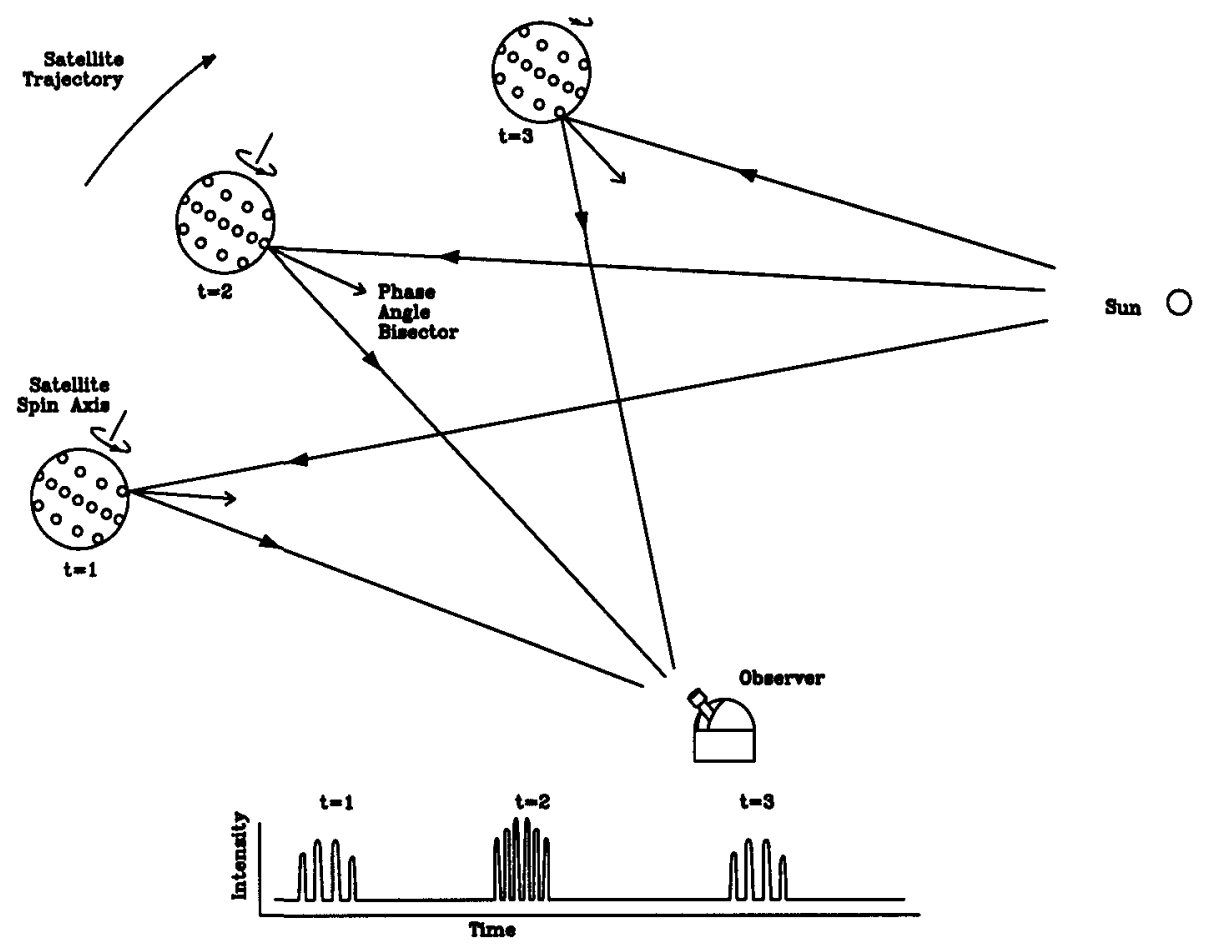

Figure 1. The position of the satellite only fulfills the Fresnel condition at certain times during the orbit. Since the angle change due to spin rate is typically faster than that due to the translation rate, we tend to see multiple flashes during the time that the Fresnel condition is fulfilled for the half-degree size of the Sun.

lying parameters can be determined by observation. At any given epoch of glint observations, the Sun and observer directions will allow glints to be seen from only one latitudinal band (Figure 1). Some minutes before and some minutes afterward, a glint or glints might be seen from a prism face in another band. One can visualize these possibilities as the result of the virtual image of the Sun moving across the spherical surface of the spacecraft and occasionally into a latitudinal belt intercepted by prism faces.

Since the spacecraft rotation remains regular in spinning (however slowly) about its initial body-fixed axis-of-rotation, or nearly so, the possible brief intervals of glint occurrences can be well-predicted by extrapolation from an earlier determination of spin-axis position and rate-of-motion. The fitting of actual glint-events to the predicted values allow differential corrections to be applied, and new predictions to be made. Observation of two successive prisms allow check of the spin period.

The scheme described requires an established starting point, and an occasional reanalysis of the observations to assure conformance to the whole history of glints and spacecraft orientation. The initial starting point used a 
TABLE 1. Solutions for the spin-axis position of LAGEOS I for given epochs.

\begin{tabular}{|c|c|c|c|c|c|c|c|c|}
\hline $\begin{array}{c}\text { Date } \\
\text { mm-dd-yy }\end{array}$ & $\begin{array}{c}\text { MJD } \\
+2440000\end{array}$ & $\begin{array}{c}\text { RA } \\
\text { [deg] }\end{array}$ & $\begin{array}{c}\text { Dec } \\
\text { [deg] }\end{array}$ & $\begin{array}{c}\mathrm{L} \\
{[\mathrm{deg}]}\end{array}$ & $\begin{array}{c}\text { W } \\
{[\mathrm{deg}]}\end{array}$ & $\begin{array}{c}\theta \\
{[\mathrm{deg}]}\end{array}$ & $\begin{array}{c}T \\
{[\mathrm{sec}]}\end{array}$ & $\begin{array}{c}d T \\
{[\mathrm{sec}]}\end{array}$ \\
\hline $09-30-88$ & 47435 & 151.87 & -79.92 & 9.76 & 0.11 & -2.43 & 37.61 & 0.40 \\
\hline $12-09-88$ & 47505 & -54.81 & -82.68 & 3.90 & 1.22 & -36.34 & 39.86 & 0.15 \\
\hline $08-23-89$ & 47762 & -15.00 & -78.00 & 1.13 & 0.03 & 40.63 & 50.12 & 0.09 \\
\hline $04-06-92$ & 48719 & -86.44 & -75.70 & 10.79 & 0.96 & 30.05 & 117.02 & 0.64 \\
\hline $05-30-92$ & 48773 & -59.16 & -77.61 & 25.45 & 1.55 & 23.47 & 122.92 & 0.69 \\
\hline $06-02-92$ & 48776 & -55.22 & -76.86 & 5.30 & 0.22 & 25.02 & 122.43 & 0.98 \\
\hline $06-10-92$ & 48784 & -52.46 & -76.43 & 31.99 & 4.08 & 40.57 & 123.9 & 1.7 \\
\hline $06-13-92$ & 48787 & -51.04 & -77.02 & 5.20 & 0.45 & 31.95 & 122.54 & 0.78 \\
\hline $07-29-92$ & 48833 & -45.68 & -80.60 & 6.23 & 0.11 & 5.54 & 128.57 & 0.79 \\
\hline $09-01-92$ & 48867 & 0.36 & -80.67 & 21.41 & 1.76 & 6.62 & 133.4 & 1.4 \\
\hline $09-29-92$ & 48896 & 20.57 & -80.49 & 22.42 & 1.16 & 18.61 & 134.6 & 1.8 \\
\hline $10-02-92$ & 48898 & 24.51 & -79.83 & 3.05 & 0.14 & 22.25 & 133.2 & 0.1 \\
\hline $10-07-92$ & 48903 & 27.95 & -79.38 & 16.64 & 1.13 & 16.58 & 134.6 & 1.4 \\
\hline $10-15-92$ & 48911 & 30.12 & -80.05 & 14.55 & 0.63 & 14.18 & 137.8 & 1.5 \\
\hline $10-23-92$ & 48919 & 38.82 & -78.94 & 12.47 & 1.31 & 9.86 & 140.5 & 5.9 \\
\hline $04-24-93$ & 49102 & 87.68 & -73.84 & 12.63 & 2.04 & 25.60 & 164.4 & 1.9 \\
\hline $04-28-93$ & 49106 & 88.98 & -72.90 & 5.41 & 1.27 & 18.75 & 166.8 & 2.3 \\
\hline $05-07-93$ & 49115 & 93.91 & -71.26 & 7.07 & 1.10 & 26.76 & 164.9 & 2.0 \\
\hline $06-02-93$ & 49141 & 89.94 & -73.54 & 5.10 & 0.66 & 12.95 & 168.54 & 0.48 \\
\hline $07-17-93$ & 49186 & 93.48 & -73.37 & 5.95 & 0.46 & 4.38 & 175.3 & 3.2 \\
\hline $09-15-93$ & 49246 & 100.82 & -72.21 & 3.89 & 2.24 & -21.81 & 185.6 & 3.7 \\
\hline $10-16-93$ & 49277 & 108.07 & -74.99 & 6.08 & 0.56 & 36.20 & 189.2 & 4.9 \\
\hline $11-11-93$ & 49303 & 114.85 & -76.47 & 29.58 & 1.24 & 14.91 & 195.2 & 2.9 \\
\hline $11-13-93$ & 49305 & 115.94 & -77.80 & 32.42 & 5.88 & 13.25 & 199.3 & 8.4 \\
\hline $02-25-95$ & 49774 & -58.71 & -66.19 & 2.61 & 0.65 & -37.37 & 312 & 33 \\
\hline $03-13-95$ & 49790 & -1.94 & -66.56 & 26.09 & 5.62 & -0.39 & 296 & 30 \\
\hline 04-05-95 & 49813 & 37.29 & -69.83 & 23.31 & 3.77 & 9.08 & 298 & 72 \\
\hline
\end{tabular}

direct method described by John Lambert (1991) which requires no extrapolation techniques when applied to photometric data when the spin-rate allows multiple glints in each of several bands.

\section{Results and Future Work}

Presented here, in Table 1, are the solutions for the spin-axis position for 28 epochs in the second decade of LAGEOS I, listing modified Julian date (MDJ), right ascention (RA), declination (Dec), solution uncertainties in terms of a rms ellipsoid of error. Also given are the inferred spin periods of the spacecraft where they are determined. 
Due to the importance of both the general geodetic use of both LAGEOS I and II, and the potential use for a future LAGEOS III, the University of Maryland is attempting to establish regular cooperative observations of the existing satellites at a variety of sites. Optimally these are performed by a tracking telescope which records the time of the glints flash to 0.2 seconds or better, although timing to 1-2 seconds proves to be useful.

Other methods, which would allow the use of additional cameras or visual observations, are under investigation and, upon establishment of these, will be propagated.

The prediction of flash times and the current status of this program will soon be available on our web page:

http://www.physics.umd.edu/rgroups/astro-metro.

\section{References}

Bertotti, B. and Iess, L.: 1991, "The rotation of Lageos", J. Geophys. Res. 96(B2), 2431. Ciufolini, I.: 1986, "Measurement of the Lense-Thirring drag on high-altitude, laserranged artificial satellites", Phys. Rev. Lett. 56, 278-281.

Ciufolini, I.: 1994, "Gravitomagnetism and status of the LAGEOS III experiment", Classical Quantum Gravity 11, A73-ff.

Currie, D.G., Kissell, K.E., Avizonis, P.V., Wellnitz, D.D.: 1995 "Remote sensing of LAGEOS I/II rotational motion", in: Scientific Exploitation of Space Surveillance Techniques, 1995 Satellite Surveillance Workshop, MIT Lincoln Laboratory, 1, 165-178.

Currie, D.G., Wellnitz, D.D., Avizonis, P., Rayner, J.D.: 1992, "LAGEOS observations and data analysis", in: Proceedings of the University of Maryland Conference on the Spin-Orbit Interactions of LAGEOS, University of Maryland, College Park.

Farinella, P., Vokrouhlicky, D., Barlier, F.: 1995, "The rotation of LAGEOS and its longterm semimajor axis decay: A self-consistent solution", J. Geophys. Res., in press.

Habib, S., Holz, D.E., Kheyfets, A., Matzner, R.A., Miller, W.A., Tolman, B.W.: 1994, "Spin dynamics of the LAGEOS satellite in support of a measurement of the Earth's gravitomagnetism", Phys. Rev. B 50(10), 6068-6079.

Johnson, C.W., Lundquist, C.A., Zurasky, J.L.: 1976, "The LAGEOS satellite", Paper presented at the XXVIIth Congress, Int. Astronaut. Fed., Anaheim, California.

Kissell, K.E.: 1992, private communication.

Lambert, J.V.: 1991, "Solving for the spin rate and the spin axis orientation of LAGEOS from photometric observations", Rockwell International Technical Note A1/550.

Ries, J., Eanes, R., Watkins, M.: 1988, "LAGEOS-III solutions and modeling", Paper presented at the LAGEOS-3 Science Advisory Group Meeting, Palo Alto, California.

Ries, J.C., Eanes, R.J., Watkins, M.M.: 1993, "Spin vector influence on LAGEOS ephemeris", Presented at the Second Meeting of IAG Special Study Group 2.130, Baltimore, MD.

Ries, J. C.: 1989, "Simulation of an experiment to measure the Lense- Thirring precession using a second LAGEOS satellite", Dissertation, University of Texas at Austin; available as CSR- 89-5, Center for Space Research, University of Texas.

Robbins, J. W., et al.: 1994, "LAGEOS I and II spin axis evolution", EOS Trans. AGU $75(16), 109$.

Rork, E.: 1996, private communication.

Rubincam, D. P.: 1990, "Drag on the LAGEOS Satellite", J. Geophys. Res. 95(B4), $4881-4886$.

Rubincam, D.P., Currie, D.G., Robbins, J.W.: 1997, "LAGEOS I once-per-revolution force due to Solar heating", Geophys. Res. Lett., accepted for publication. 